\title{
Den Erfolg behindern nicht die Holzschuhe
}

Hintergrund und Fragestellungen: Prävention des Diabetes mellitus Typ 2 ist möglich. Das hat eine Vielzahl von wissenschaftlichen Studien gezeigt. Auf der anderen Seite scheitern wir nahezu komplett, dieses Wissen in unseren Praxisalltag zu übersetzen. Woran liegt das? Was macht es so schwer, eine Verhaltensänderung bei Patienten zu initiieren? Ist der Patient ,schuld“ oder nutzen wir die falschen Interventionsstrategien? Welche anderen Ursachen könnten dafür vorliegen? Um einen Teil dieser Fragen zu beantworten, wurde von Arlette Hesselink in Holland die folgende Studie durchgeführt.

Patienten und Methoden: In eine clusterrandomisierten klinischen Studie wurden 366 Teilnehmer aus 26 Primary-Care-Praxen in den Niederlanden einbezogen. Bei den Patienten war eine gestörte Nüchternglukose diagnostiziert worden. Sie waren motiviert, ihren Lebensstil zu ändern. 197 Personen waren

\section{Originalie}

Hesselink AE, Rutten GE, Slootmaker SM et al. Effects of a lifestyle program in subjects with Impaired Fasting Glucose, a pragmatic cluster-randomized controlled trial. BMC Fam Pract. 2015;16(1):183. in der Interventionsgruppe und 169 in der Gruppe, die eine herkömmliche Betreuung bekam. Die Nachbeobachtung dauerte 2 Jahre. Die Interventionsgruppe erhielt hatte 5-6mal im ersten Jahr beratenden Kontakt mit einer Schwester in den genann-

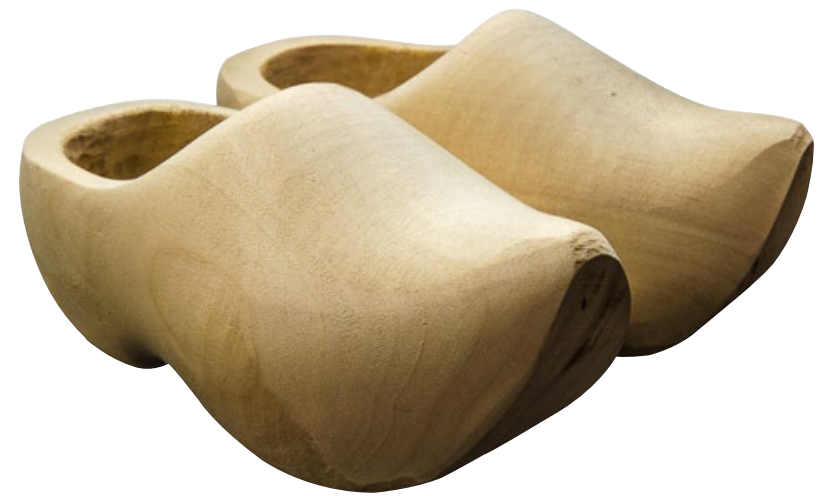

ten Praxen, die andere nur einmal pro Jahr. Ziel dabei war eine Verbesserung der körperlichen Alltagsaktivität und des Ernährungsverhaltens. Als Outcome in der Studie wurde Body-MassIndex (BMI) und Alltagsaktivität gewertet.

Ergebnisse: Die beiden Studiengruppen zeigten nach 1 und 2 Jahren keine/nur geringe Differenzen hinsichtlich des BMI. Interessant war aber, dass die Personen in der Interventionsgruppe im Alltag deutlich aktiver waren und ein großer Teil es geschafft hat, 30 Minuten pro Tag körperlich aktiv zu sein. Nach einem Jahr schafften das etwa viermal so viele und nach zwei Jahren immer noch doppelt so viele in der Interventions- wie in der Kontrollgruppe. Interessant war auch, dass die Teilnehmer der Interventionsgruppe meist weniger Beratungen erhielten, als im Protokoll vorgeschrieben war, das aber von ihnen immer noch als zu aufwendig empfunden wurden.

Schlussfolgerungen: Die Autoren schlussfolgern, dass dieses relativ einfache Programm bei Personen mit einer gestörten Nüchternglukose deutliche Verbesserungen bei der körperlichen Alltagsaktivität ergeben hat, aber nicht beim BMI. Manko ist laut Autoren, dass selbst diese minimale Intervention von den Teilnehmern als noch zu aufwendig empfunden wurde.

\section{- Kommentar von Prof. Dr. med. Peter Schwarz}

\section{Viele Aktivitäts-Barrieren, aber auch Hoffung}

Die Studie erscheint sehr einfach, beinhaltet aber einige interessante Aspekte für die Versorgungsrealität in Deutschland. In Holland wurde als Intervention eine mehrmalige Konsultation einer Schwester aus der Arztpraxis gewählt. Vergleicht man das in Deutschland, entspräche das den Interventionsprogrammen, die wir nach §20, SGB 5 anwenden können. Für die meisten Bundesländer sind da sogar bis zu 8 Termine möglich. In der Studie ging es um Personen mit einer gestörten Nüchternglukose (IFG). Diese Personen würden klassischerweise in Deutschland unter $\S 20$, SGB 5 fallen. Weiterhin konnte gezeigt werden, dass die Intervention bei den Probanden keine BMI-veränderung, aber dafür eine bis zu vierfache Verbesserung des Grades an Alltagsaktivität erreicht hat. Das ist toll. Es erscheint als ein suffizienter Weg, Diabetes zu verhindern, wenn der Grad Alltagsaktivität signifikant verbessert wird. Das konnte erreicht werden, obwohl die vorgesehenen Konsultationen bei vielen nicht alle erreicht wurden. Wenn man dieses Vorgehen besser strukturiert, lässt sich hier vielleicht noch eine viel stärkere Aktivierung zu mehr körperlicher Alltagsaktivität realisieren. Als dritten Schwerpunkt zeigte sich aber, dass dieses Vorgehen von den Patienten als sehr aufwendig empfunden wird. Dieses Ergebnis ist vermutlich für einen Teil der eingangs dargestellten Problematik verantwortlich. Eine Schulung oder Motivation zu einem gesünderen Lebensstil wird häufig nicht als nötige medizinische Maßnahme und als aufwendig empfunden. Das ist eine Barriere, die schwer zu durchbrechen ist. So lange Gesundheit als etwas betrachtet wird, was von außen über den Arzt realisiert wird, wird man diese Barriere kaum durchbrechen können. Ein Selbstverständnis, dass jeder seine Gesundheit täglich selbst erwirtschaftet, könnte etwas ändern - wäre aber ein Paradigmenwechsel in der Sichtweise.

Die Studie macht Hoffnung und zeigt Barrieren. Wenn wir das holländische Vorgehen als „Präventionsprodukt" verstehen, finden sich dafür aber nur wenige „Käufer“. Mit dem Dilemma müssen wir leben und versuchen, getriggert durch Studien wie diese erfolgreiche Präventionsprodukte zu entwickeln.

Prof. Dr. med. Peter Schwarz, MBA - Universität Dresden 